\title{
LARGE-SCALE STRUCTURE AMONG ABELL CLUSTERS AND COMPARISONS TO MODELS
}

\author{
David J. Batuski \\ Space Telescope Science Institute, Homewood Campus \\ Baltimore, MD 21218 USA \\ Jack O. Burns \\ Institute for Astrophysics, Univ. of New Mexico \\ Albuquerque, NM 87131 USA \\ Adrian Melott \\ Department of Physics and Astronomy, Univ. of Kansas \\ Lawrence, KS 66045 USA
}

In a continuing redshift observation program, we have recently determined or confirmed the redshifts of $32 R \geq 0$ Abell clusters. With these data added to the previously measured cluster redshifts, there is now a sample of Abell clusters to $z=0.085$ that is $92 \%$ complete in redshift measurements. The sample consists of 225 clusters in the North and South Galactic Caps, with latitude $|b|>30^{\circ}$ to minimize obscuration effects. The longitude range $270^{\circ}<l<360^{\circ}$ was also excluded for this sample because of an apparent large spur of galactic absorption.

With these most recent redshift data, we calculated a new two-point correlation function for the $R \geq 0$ Abell clusters, which shows indications of structure on scales as large as $300 \mathrm{Mpc}\left(H_{0}=50 \mathrm{~km} \mathrm{sec}^{-1} \mathrm{Mpc}^{-1}\right)$. In particular, there appears to be a sizeable "bump" in the function around $300 \mathrm{Mpc}$.

The amount of structure present in this sample of Abell clusters has been compared with numerical supercomputer simulations (with $64^{3}$ particles) of the isothermal (IS), neutrino (NE), and cold particle (CP) models for large-scale structure, assuming a flat universe and $H_{0}=50,75$, and 100 . High-density clusters of particles were identified in each simulation. Correlation and percolation tests were then used to compare the spatial distribution of these high-density points with the apparent superclustering among Abell clusters. While $H_{0}=50$ gave the best results, none of these models came very close to matching the observations. The NE model appears to form many superclusters, each consisting of a few clusters very densely spaced, quite unlike Abell cluster superclusters with many $(>10)$ member clusters and large spatial extents. The CP and IS models have too many comparatively isolated clusters, and thus show only weak superclustering effects.

We also generated two simulations of the CP model with $\Omega=0.5$, and $\Omega=0.2$, to see if the lower density would cause sufficiently large structure to match the observations. These simulations did indeed contain more clusters with relatively small separations from neighboring clusters (i.e., more apparent superclusters), but these groupings were still not sufficiently large to match the Abell cluster superclusters to within $2 \sigma$ errors in one of our tests.

J. Audouze et al. (eds.), Large Scale Structures of the Universe, 543.

(C) 1988 by the IAU. 